\title{
Channeling of charge carrier plasmons in carbon nanotubes
}

\author{
C. Kramberger, ${ }^{1}$ F. Roth,${ }^{2}$ R. Schuster, ${ }^{2}$ R. Kraus, ${ }^{2}$ M. Knupfer,${ }^{2}$ E. Einarsson, ${ }^{1}$ S. Maruyama, ${ }^{1}$ \\ D. J. Mowbray, ${ }^{3}$ A. Rubio, ${ }^{3,4}$ and T. Pichler ${ }^{5}$ \\ ${ }^{1}$ Department of Mechanical Engineering, The University of Tokyo, 7-3-1 Hongo, Bunkyo-ku, Tokyo JP-113-8656, Japan \\ ${ }^{2}$ IFW Dresden, Helmholtzstraße 20, DE-01069 Dresden, Germany \\ ${ }^{3}$ Nano-Bio Spectroscopy Group and ETSF Scientific Development Centre, Departamento de Física de Materiales, Universidad del País \\ Vasco, Centro de Física de Materiales CSIC-UPVIEHU-MPC and DIPC, Avenida de Tolosa 72, ES-20018 San Sebastián, Spain \\ ${ }^{4}$ Fritz-Haber-Institut der Max-Planck-Gesellschaft, DE-14195 Berlin, Germany \\ ${ }^{5}$ University of Vienna, Faculty of Physics, Strudlhofgasse 4, AT-1090, Vienna, Austria
}

(Received 24 August 2011; revised manuscript received 30 January 2012; published 16 February 2012)

\begin{abstract}
$A b$ initio calculations of the loss function of potassium-intercalated and electron-loaded bundles of single-walled carbon nanotubes yield a channeled-charge-carrier plasmon without perpendicular dispersion. Experimentally, we probe the momentum-dependent loss function of thin bundles consisting of only a few potassium-intercalated single-walled carbon nanotubes by angle-resolved electron-energy-loss spectroscopy and confirm this intrinsic channeling. The charge-carrier-plasmon energy is via in situ intercalation and is tunable in the near-visible infrared-energy range from 0.85 to $1.15 \mathrm{eV}$.
\end{abstract}

DOI: 10.1103/PhysRevB.85.085424

PACS number(s): 73.20.Mf, 73.22.-f, 78.20.Bh

\section{INTRODUCTION}

In conventional bulk metallic systems the collective electronic-excitation spectrum is comprised of free-chargecarrier or intraband plasmons and interband plasmons. Plasmons are, in analogy to sound waves, longitudinal density fluctuations that propagate through the electron plasma. In the optical limit at vanishing momenta $q=\hbar k \rightarrow 0$, the metallic intraband plasmon scales with the electron density $n_{e}$ and occurs at $\omega_{P}^{2}=n_{e} e^{2} / m^{\star} \varepsilon_{0}$. Staged graphite-intercalation compounds (GICs) are a special case of metallic systems with well-defined stoichiometry. The intraband plasmon as well as the interband plasmon of the electronic $\pi$ and $\sigma$ bands in GICs have been known for a long time. ${ }^{1}$

Another important classification of plasmons is the distinction between bulk and surface modes. The latter are confined to the interface of two dielectric media where the sign of the frequency-dependent dielectric response $\varepsilon(\omega)$ changes. For a Drude metal in vacuum, the free surface plasmon is found at $\omega_{S}=\omega_{P} / \sqrt{2}$. In a free-charge-carrier plasma there is a quadratic dispersion relation ${ }^{2}$ whereas the intra- and interband-plasmon dispersion of actual solids is influenced by the band structure. ${ }^{3,4}$ The two-dimensional domain of a surface plasmon cancels any defined momentum state or dispersion relation in the perpendicular direction. Well-defined momentum states only apply parallel to the surface.

Individual single-walled carbon nanotubes (SWNTs) or thin wires thereof are archetypes of quantum wires with unique and strongly anisotropic dielectric properties. Macroscopically aligned SWNT films ${ }^{5-7}$ may be envisaged as a nano-meshed metamaterial. In dielectric metamaterials the plasmon response may also be affected by crystal local-field effects (LFEs). These are of more pronounced importance in isolated sheets or even nanowires. ${ }^{8-11}$ The full electronicexcitation spectrum beyond the optical limit is accessible by electron-energy-loss spectroscopy (EELS). ${ }^{7}$ Angle-resolved EELS (AR-EELS) assesses plasmon energies and dispersion relations. ${ }^{12,13}$
The present work addresses the frequency- and momentumdependent dielectric response of freestanding metallic nanowires. Our $a b$ initio calculations on K-intercalated- and electron-loaded-SWNT bundles show a channeled low-energy charge-carrier plasmon. The electron density and hence the frequency of the charge-carrier plasmon in freestanding SWNTs is tuned by in situ potassium intercalation. We confirm the presence of a channeled and tuneable charge-carrier plasmon in the near-infrared region from 0.85 to $1.15 \mathrm{eV}$ by AR-EELS measurements.

\section{METHODS}

Density-functional-theory (DFT) calculations for bulkSWNT bundles were performed using the real-space projectoraugmented-wave (PAW) code GPAW ${ }^{14}$ with the Perdew-BurkeErnzerhof (PBE) exchange-correlation (xc) functional. ${ }^{15}$ The optimized unit cells for bundles of $(10,10)[(17,0)]$ SWNTs of 2.470 (4.272) $\AA$ in the axial direction and 16.95 (16.88) $\AA$ between SWNTs in the bundle cross section were employed, containing 40 (68) carbon atoms and 2 potassium atoms, respectively. Atomic coordinates were relaxed until forces were below $0.05 \mathrm{eV} / \AA$. Charged calculations with $Q=$ $-1,-2, \ldots,-8 e /$ cell were performed with a uniform background potential to eliminate interactions between neighboring unit cells. To determine the effect of lattice expansion due to charging of the SWNT bundles, calculations for the highest considered electron loading $e / \mathrm{C}=12.5 \%$ were performed by expanding the lattice parameters in the bundle cross section by $1 \%, 2 \%, \ldots, 10 \%$. Even a $10 \%$ lattice expansion of the SWNT bundle did not noticeably affect the charge-carrier-plasmon energy. Note that the uncharged geometries were further optimized within the electron-loaded model. A MonkhorstPack $k$-point sampling with $25(15) k$ points along the axial direction and $4 \times 4 k$ points in the bundle cross section was employed, yielding a momentum-transfer resolution $\Delta \mathbf{q}$ of $\approx 0.1 \AA^{-1}$. Loss-function calculations were performed using linear-response time-dependent DFT within the random-phase approximation ${ }^{16}$ with LFEs included. 
Samples for AR-EELS are freestanding sparse films of vertically aligned SWNTs, which were prepared as described in Ref. 17. The individual nanowires are composed of typically five to seven SWNTs and are arranged in a sparse random array with a bulk density as low as $\rho \approx 0.05 \mathrm{~g} \mathrm{~cm}^{-3} \cdot{ }^{18,19}$ The diameters of the individual SWNTs are $\sim 2 \mathrm{~nm}$. Commercial potassium getters from SAES getters were used to evaporate potassium and perform successive in situ intercalation of the sparse-SWNT film. The angle-resolved loss function of potassium-intercalated sparse-SWNT films was measured in a purpose-built EELS spectrometer, ${ }^{20}$ operated at energy and momentum resolutions of $80 \mathrm{meV}$ and $0.03 \mathrm{AA}^{-1}$, respectively. The incident beam is in normal transmission parallel to the net alignment direction, and the momentum transfer $q$ is predominantly perpendicular to the axis of the sparse SWNTs.

\section{RESULTS}

The loss function of GIC $\mathrm{KC}_{8}$, fully intercalated bulk SWNTs, and fully intercalated sparse SWNTs are shown in Fig. 1. All three potassium-intercalated allotropes of $s p^{2}$ carbon exhibit a charge-carrier plasmon at small energies (•), an interband excitation of the electronic $\pi$ system at intermediate energies $(\pi)$, and lastly a more structured $\pi+\sigma$ interband response of the remaining $\mathrm{C} 2 p$ electronic system $(\star)$. The atomic $\mathrm{K} 3 p \rightarrow \mathrm{K} 4 s$ excitation $(\mathrm{K})$ is found embedded in the $\pi+\sigma$ structures. The most apparent observation is that all charge-carrier and interband plasmons are downshifted when going from $\mathrm{KC}_{8}$ via bulk-SWNT bundles to sparse SWNTs. This behavior is entirely conceivable within the Drude-Lorentz model in which the longitudinal plasma frequency $\omega_{L}$ is offset to the transversal (optical) plasma frequency $\omega_{T}$ according to $\omega_{L}^{2}=\omega_{T}^{2}+\omega_{P}^{2}$. The smaller density results in a lower $\omega_{P}$. The additional electrons are provided by potassium counterions, whose atomic concentrations are derived from the $\mathrm{C} 1 s$ and

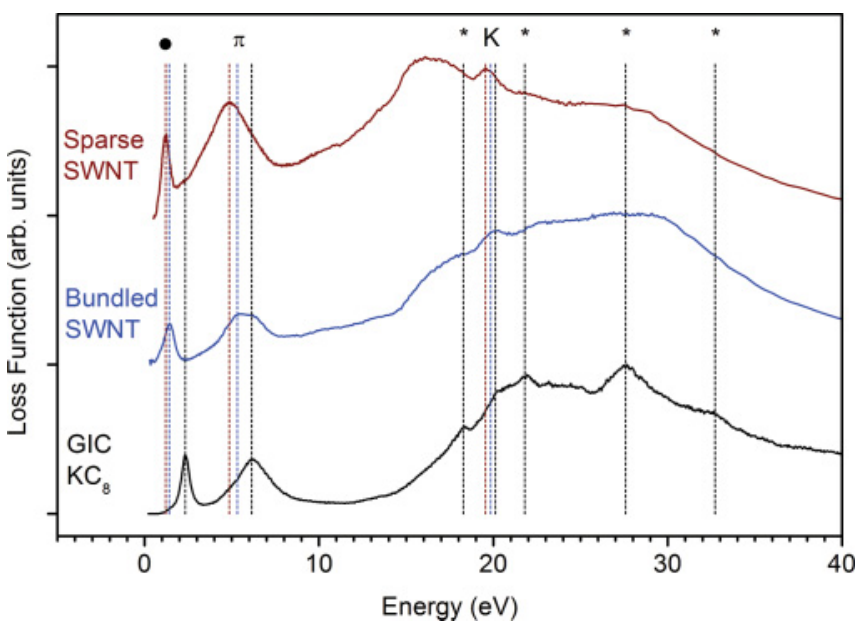

FIG. 1. (Color online) The loss functions of fully $n$-doped allotropes of $s p^{2}$ carbon networks. The loss functions were recorded at a momentum transfer $q=0.1 \AA^{-1}$. Vertical lines compare the positions of plasmon modes in the three different samples. $\bullet$ denotes the charge-carrier plasmon, $\mathrm{K}$ the atomic $\mathrm{K} 3 p \rightarrow \mathrm{K} 4 s$ transition, $\pi$ the interband plasmon of the electronic $\pi$ system, and $\star$ the multiple oscillators of the $\pi+\sigma$ electrons.
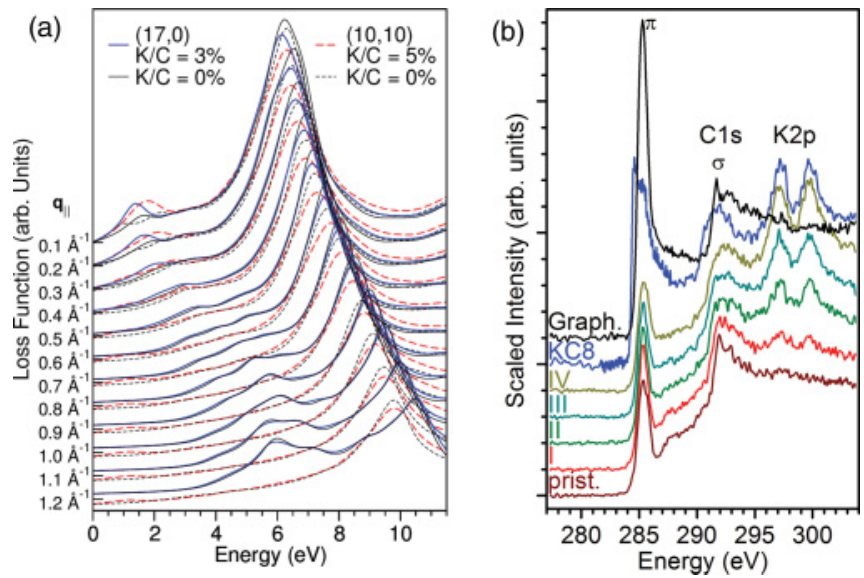

FIG. 2. (Color online) (a) Calculated loss functions of pristine and K-intercalated bulk-SWNT bundles for $q_{\|}$. (b) Core-level loss edges of the $\mathrm{C} 1 s$ and $\mathrm{K} 2 p$ in graphite, $\mathrm{KC}_{8}$, and doped and pristine sparse SWNTs.

K $2 p$ core-level excitations shown in Fig. 2. The different densities, observed peak positions, and widths of the intraband plasmon at the individual doping steps are collected in Table I.

The changes in the loss function during successive potassium intercalation of the nanowires are shown in Fig. 3. The vertical dashed line marks the unchanged position of the $\pi$ plasmon. The intraband plasmon $(\bullet)$ is gradually upshifted with increasing potassium concentrations. The square of the plasmon position $\omega_{P}^{2}$ is plotted against the potassium concentration in Fig. 3(b). Our current studies on sparse SWNTs are compared to pioneering studies on graphite-intercalation compounds $^{1}$ as well as preceding studies on SWNT bundles. ${ }^{21}$ All three $s p^{2}$-carbon systems exhibit a monotonic increase of $\omega_{P}^{2}$ with increasing $\mathrm{K} / \mathrm{C}$ ratio. A key difference here is the sharp onset of an intraband plasmon near a $\mathrm{K} / \mathrm{C}$ of $4-5 \%$ in SWNT bundles as opposed to the smooth behavior in the sparse-SWNT films. In SWNT bundles the intraband plasmon occurs only when the semiconducting SWNTs are driven metallic as well. ${ }^{22}$ We suggest that the competition for charge transfer only occurs inside interstitial channels where semiconducting and metallic SWNTs meet. Neither the calculated all-metallic or semiconducting bundles nor the surface-dominated sparse SWNTs provide such interstitial

TABLE I. Potassium concentrations and intraband-plasmon energies $\omega_{P}$ for a sparse-SWNT film in comparison to those of fully intercalated bundled SWNTs and $\mathrm{KC}_{8}$ from Ref. 21. The subscripts $B$ and $S$ denote bulk and surface plasmons, respectively.

\begin{tabular}{lccccc}
\hline \hline Step & $\mathrm{K} / \mathrm{C}(\%)$ & $\rho\left(\mathrm{g} \mathrm{cm}^{-3}\right)$ & $\omega_{P}(\mathrm{eV})$ & $\varepsilon_{\infty}$ & $\Gamma(\mathrm{eV})$ \\
\hline pristine & & $\sim 0.05$ & & 1 & \\
I & 1.6 & $\sim 0.05$ & $0.85_{S}$ & 1 & 0.5 \\
II & 5.5 & $\sim 0.05$ & $1.05_{S}$ & 1 & 0.5 \\
III & 8.4 & $\sim 0.05$ & $1.10_{S}$ & 1 & 0.5 \\
IV & 12.5 & $\sim 0.05$ & $1.15_{S}$ & 1 & 0.5 \\
bundled & 12.5 & 1.3 & $1.4_{B}$ & 6.1 & 0.7 \\
GIC $\mathrm{KC}_{8}$ & 12.5 & 2.3 & $2.4_{B}$ & 6.7 & 0.2 \\
\hline \hline
\end{tabular}



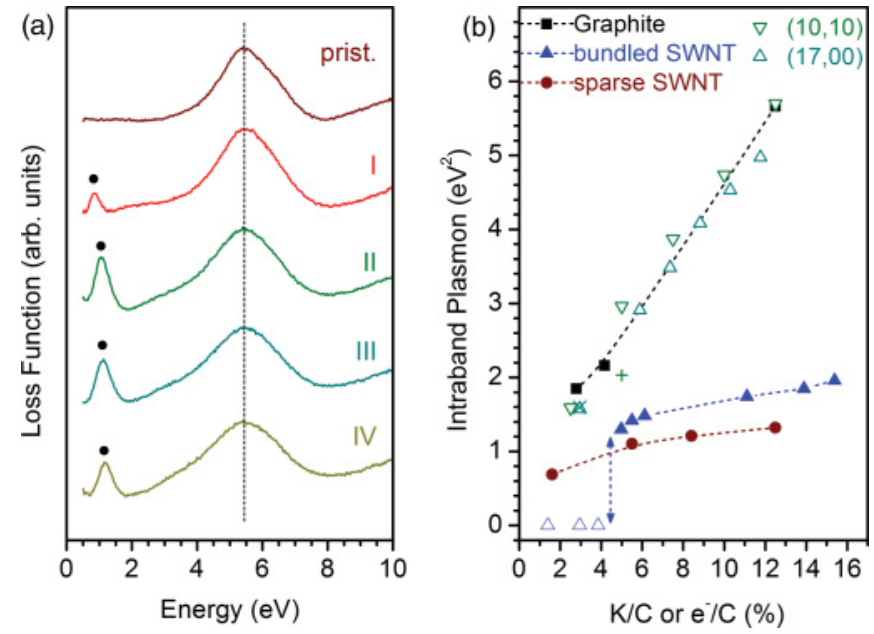

FIG. 3. (Color online) (a) Low-energy range of the loss functions of successive potassium-intercalated sparse SWNTs. (b) Square of the peak position of the charge-carrier plasmon versus potassium concentration for graphite (squares), SWNT bundles (up triangles), and sparse SWNTs (circles) in comparison to calculations of electronloaded (open, down, and up triangles) and K-intercalated $(+, \times)$ bundles of all $(10,10)$ and all $(17,0)$ SWNTs, respectively. Dotted lines are guides for the eye.

sites. Therefore, neither the calculated $(10,10)$ or $(17,0)$ SWNT bundles nor sparse-SWNT films show this sharp onset.

The empirical data on bundled and sparse SWNTs in Fig. 3(b) is clearly beyond the classical homogenous Drude model with complete charge transfer, which would feature a straight line. In contrast, the calculated charge-carrier plasmon in electron-loaded all- $(10,10)$ or $(17,0)$ bundles lies close to the interpolated data from intercalated graphite. It also scales linearly with the charge transfer.

The comparison of electron-loaded and potassiumintercalated calculations suggests that there is a complete charge transfer in GICs as well as $\mathrm{K}$-intercalated semiconducting SWNTs but only a fractional $(\sim 2 / 3)$ charge transfer in K-intercalated metallic SWNTs. The very different changes of the $\pi$ resonance of the $\mathrm{C} 1 s$ core edges in Fig. 2 of $\mathrm{KC}_{8}$ and $\mathrm{K}$-intercalated nanowires underpins this possibility. On this basis, we propose that on the nanowires, $\mathrm{K}$ ions will first occupy the fully coordinated sites and only later lower-coordinated sites with reduced partial-charge transfers. Thus the increase in $\omega_{P}^{2}$ levels off, and its energy is finely tuned by the $\mathrm{K}$ concentration.

Regarding the different plasmon energies in sparse SWNTs and bulk bundles, the sparse SWNTs form thin bundles that resemble the textbook example of a metamaterial consisting of parallel metallic cylinders. The key characteristics of such a classical system ${ }^{23}$ with a material-intrinsic $\omega_{P}$ but only a very low filling ratio $f \ll 1$ are (i) a bulk on-axis plasmon that scales with the filling ratio $f \omega_{P}$ and (ii) an orthogonal localized surface plasmon at $\omega_{P} / \sqrt{1+\varepsilon_{\infty}}$. In the present case of negligible dielectric screening, $\varepsilon_{\infty}$ becomes unity, and the surface plasmon arises at $\omega_{P} / \sqrt{2}$. The low filling ratio of $f \approx 0.07$ switches off the on-axis plasmon, but the orthogonal surface mode is preserved.
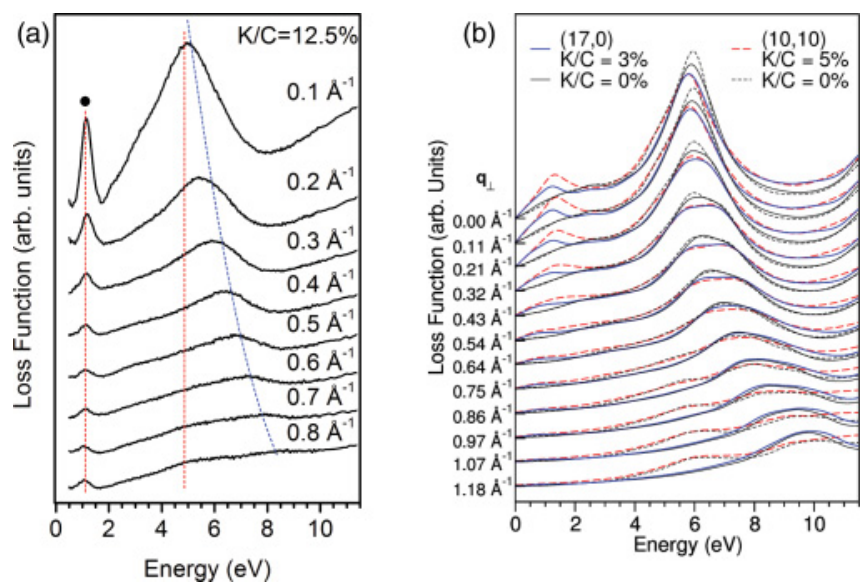

FIG. 4. (Color online) (a) AR-EELS of sparse K-intercalated SWNTs. The momentum transfer $q$ is perpendicular to the net alignment. (b) Calculated loss functions of pristine and $\mathrm{K}$-intercalated bulk-SWNT bundles for $q_{\perp}$.

In this intuitive model we can interpret the down-scaled intraband plasmon in nanowires as a localized surface mode of the charge-carrier plasmon in bulk bundles. The scaling of the curves in Fig. 3 yields a scaling factor of $\omega_{P B}^{2} / \omega_{P S}^{2} \sim 4 / 3$ in sparse SWNTs.

The momentum dispersion of the loss function in Fig. 4(a) reveals the elementary electronic-excitation spectrum beyond the optical limit $(q \rightarrow 0)$. The dotted lines highlight the dispersion of individual features. We find a pair of one nondispersive and one dispersive plasmon for the $\pi$-interband plasmon. ${ }^{11}$ The charge-carrier plasmon is observed exclusively as a nondispersive mode. The calculations of the loss functions in Fig. 4(b) were performed for pristine- and K-intercalatedSWNT bundles. The low-energy range shown here features the $\pi$ plasmon as well as a new charge-carrier plasmon above $1 \mathrm{eV}$ specific to intercalated bundles. Calculations for $q_{\|}$are shown in Fig. 2. Here the additional charge-carrier response shows dispersive behavior for the lowest momentum transfers before it is quickly dampened by interband transitions. The comparison of $q_{\|}$and $q_{\perp}$ reveals the exclusive appearance of the nondispersive $\pi$ plasmon for $q_{\|}$in $(17,0)$ and $q_{\perp}$ in $(10,10)$. The nondispersive $\pi$ plasmon is therefore always found for $q$ along the armchair direction. ${ }^{24}$ The charge-carrier plasmon in K-intercalated armchair and zigzag bundles is found to be independent of $q$ being along the armchair or zigzag direction but behaves differently for $q_{\|}$and $q_{\perp}$.

While the charge-carrier plasmon behaves ordinarily for $q_{\|}$, the gradual damping and absence of orthogonal dispersion with $q_{\perp}$ are unexpected in a bulk system. They are the hallmark of one-dimensional channeling on individual SWNTs inside a bulk bundle.

As counterintuitive as this ab initio finding might seem, it is fully corroborated by no less unexpected experimental findings. ${ }^{21}$ The current AR-EELS experiments demonstrate a doping-dependent and non- $q_{\perp}$-dispersive intraband plasmon in sparse SWNTs. The ab initio calculations on intercalated and electron-loaded bundles provide an $a b$ initio footing for the interpretation of the observed plasmon as the charge-carrier response in effectively decoupled SWNTs. This interpretation 
also explains the nondispersive behavior of the charge-carrier plasmon observed in K-intercalated bulk-SWNT bundles. ${ }^{21}$

Another very important aspect of one-dimensional electronic systems is the electronic Tomonaga liquid. ${ }^{25}$ It was first evidenced on mixed-SWNT material by the characteristic power-law onset in photoemission spectra. ${ }^{26}$ It was believed that $2 / 3$ of semiconducting SWNTs act as spacers to maintain one-dimensional metallic channels inside the bulk bundles. Later, it was discovered that all metallic-SWNT bundles are a Tomanaga liquid as well. ${ }^{27}$ The latter surprising finding is fully in line with our notion that metallic SWNTs in a bundle do act as independent channels. The channeling of the intraband plasmon is not only provided by the nanowire morphology, but it is indeed an intrinsic property of each and every SWNT.

\section{CONCLUSIONS}

Summarizing, we have demonstrated direct experimental access to the full spectrum of localized and delocalized interband and charge-carrier plasmons in bulk and on sparse
SWNTs. We have also put fractional charge transfer in alkali-intercalated-SWNT bundles as well as the intrinsic nature of channeled-charge-carrier plasmons in SWNTs on an $a b$ initio footing. The intrinsic local channeling gives rise to uniform and well-defined bulk properties, independent of the detailed morphology. This robustness against variations in the microscale is a versatile aspect for plasmonic devices based on carbon nanotubes.

\section{ACKNOWLEDGMENTS}

We acknowledge funding by DFG Grant No. PI 440/4, European Research Council Advanced Grant DYNamo (ERC-2010-AdG Proposal No. 267374), Spanish MICINN (Grant No. FIS2010-21282-C02-01), Grupos Consolidados UPV/EHU del Gobierno Vasco (Grant No. IT-319-07), ACI-Promociona (Grant No. ACI2009-1036), and European Community e-I3 ETSF project (Contract No. 211956). C.K. acknowledges the Austrian Academy of Science (Grant No. 11456). D.J.M. acknowledges funding through the Spanish Juan de la Cireva Program (Grant No. JCI-2010-08156).
${ }^{1}$ J. J. Ritsko, E. J. Mele, and I. P. Gates, Phys. Rev. B 24, 6114 (1981)

${ }^{2}$ J. Lindhard, Kgl. Dan. Vidensk. Selsk. Mat. Fys. Medd. 28, 1 (1954).

${ }^{3}$ P. Nozieres and D. Pines, Phys. Rev. 113, 1254 (1959).

${ }^{4}$ H. Ehrenreich and M. H. Cohen, Phys. Rev. 115, 786 (1959).

${ }^{5}$ Y. Murakami, S. Chiashi, Y. Miyauchi, M. H. Hu, M. Ogura, T. Okubo, and S. Maruyama, Chem. Phys. Lett. 385, 298 (2004).

${ }^{6}$ Y. Murakami, E. Einarsson, T. Edamura, and S. Maruyama, Phys. Rev. Lett. 94, 087402 (2005).

${ }^{7}$ O. Stéphan, D. Taverna, M. Kociak, K. Suenaga, L. Henrard, and C. Colliex, Phys. Rev. B 66, 155422 (2002).

${ }^{8}$ S. L. Adler, Phys. Rev. 126, 413 (1962).

${ }^{9}$ N. Wiser, Phys. Rev. 129, 62 (1963).

${ }^{10}$ G. Onida, L. Reining, and A. Rubio, Rev. Mod. Phys. 74, 601 (2002).

${ }^{11}$ C. Kramberger, R. Hambach, C. Giorgetti, M. H. Rümmeli, M. Knupfer, J. Fink, B. Büchner, L. Reining, E. Einarsson, S. Maruyama, F. Sottile, K. Hannewald, V. Olevano, A. G. Marinopoulos, and T. Pichler, Phys. Rev. Lett. 100, 196803 (2008).

${ }^{12}$ T. Pichler, M. Knupfer, M. S. Golden, J. Fink, A. Rinzler, and R. E. Smalley, Phys. Rev. Lett. 80, 4729 (1998).

${ }^{13}$ M. Knupfer, T. Pichler, M. S. Golden, J. Fink, A. Rinzler, and R. E. Smalley, Carbon 37, 733 (1999).

${ }^{14}$ J. Enkovaara, C. Rostgaard, J. J. Mortensen, J. Chen, M. Dułak, L. Ferrighi, J. Gavnholt, C. Glinsvad, V. Haikola, H. A. Hansen, H. H. Kristoffersen, M. Kuisma, A. H. Larsen, L. Lehtovaara, M. Ljungberg, O. Lopez-Acevedo, P. G. Moses, J. Ojanen, T. Olsen, V. Petzold, N. A. Romero, J. Stausholm-Møller, M. Strange, G. A. Tritsaris, M. Vanin, M. Walter, B. Hammer, H. Häkkinen, G. K. H. Madsen, R. M. Nieminen, J. K. Nørskov, M. Puska, T. T. Rantala,
J. Schiøtz, K. S. Thygesen, and K. W. Jacobsen, J. Phys.: Condens. Matter 22, 253202 (2010).

${ }^{15}$ J. P. Perdew, K. Burke, and M. Ernzerhof, Phys. Rev. Lett. 77, 3865 (1996).

${ }^{16}$ J. Yan, J. J. Mortensen, K. W. Jacobsen, and K. S. Thygesen, Phys. Rev. B 83, 245122 (2011).

${ }^{17}$ Y. Murakami and S. Maruyama, Chem. Phys. Lett. 422, 575 (2006).

${ }^{18}$ H. M. Duong, E. Einarsson, J. Okawa, R. Xiang, and S. Maruyama, Jpn. J. Appl. Phys. 47, 1994 (2008).

${ }^{19}$ E. Einarsson, H. Shiozawa, C. Kramberger, M. H. Rummeli, A. Gruneis, T. Pichler, and S. Maruyama, J. Phys. Chem. C 111, 17861 (2007).

${ }^{20}$ J. Fink, Adv. Electron. Electron Phys. 75, 121 (1989).

${ }^{21}$ X. Liu, T. Pichler, M. Knupfer, and J. Fink, Phys. Rev. B 67, 125403 (2003).

${ }^{22}$ C. Kramberger, H. Rauf, M. Knupfer, H. Shiozawa, D. Batchelor, A. Rubio, H. Kataura, and T. Pichler, Phys. Rev. B 79, 195442 (2009).

${ }^{23}$ T. McNeish, G. Gumbs, and A. Balassis, Phys. Rev. B 77, 235440 (2008).

${ }^{24}$ S. Dmitrović, T. Vuković, B. Nikolić, M. Damnjanović, and I. Milošević, Phys. Rev. B 77, 245415 (2008).

${ }^{25}$ S. Tomonaga, Prog. Theor. Phys. 5, 544 (1950).

${ }^{26}$ H. Ishii, H. Kataura, H. Shiozawa, H. Yoshioka, H. Otsubo, Y. Takayama, T. Miyahara, S. Suzuki, Y. Achiba, M. Nakatake, T. Narimura, M. Higashiguchi, K. Shimada, H. Namatame and M. Taniguchi, Nature (London) 426, 540 (2003).

${ }^{27}$ P. Ayala, Y. Miyata, K. De Blauwe, H. Shiozawa, Y. Feng, K. Yanagi, C. Kramberger, S. R. P. Silva, R. Follath, H. Kataura, and T. Pichler, Phys. Rev. B 80, 205427 (2009). 